Karol Górski*

\title{
EINE DEUTSCH-POLNISCHE, POETISCHE AUSEINANDERSETZUNG ZUM AUFTAKT \\ DER NÄCHSTJÄHRIGEN FEIERLICHKEITEN ANLÄSSLICH \\ DES 100. JAHRESTAGES DES AUSBRUCHS \\ DES GROSSPOLNISCHEN AUFSTANDES 1918/19 \\ IN DER PREUSSISCHEN PROVINZ POSEN
}

Zum Ende 2018 wird sich zum 100. Mal der Jahrestag des Ausbruchs des Großpolnischen Aufstandes 1918/19 in Posen, der Hauptstadt der preußischen Provinz Posen jähren. Zu diesem gloriareichen Jubiläum wird man vor Ort auf offizieller Ebene, allerdings wohl immer weniger im Familiengedächtnis, der Opfer der polnischen patriotischen Tat vor 100 Jahren, vor allen Dingen aber des Ruhmes der großpolnischen Aufständischen gedenken. Es wird gefeiert werden, es werden Kränze mit weiß-roten Schärpen niedergelegt werden, an den Denkmälern werden die Fahnen im Winde flattern, schlagen die Trommeln und wird der Eid des ewigen Gedächtnisses der polnischen Erhebung in der Provinz Posen aus der Jahreswende 1918/19 auf die staatlichen und aufständischen Fahnen geschworen. Für die Zwecke einer besseren Rezeption des Jahrestages durch die jüngere Generation wurde inzwischen schon zum 96. Begehen dessen sogar ein entsprechend thematisierter Film ${ }^{1}$ in einem einzigartigen, okkult eingefärbten Aufzug in polnischen Kinos in großer Aufmachung präsentiert. Die zu diesem Anlass erwartete Stimmung wohnt auch einer lyrischen Vorankündigung des feierlichen Geschehens in Form des von dem Autor dieser Abhandlung dazu verfassten Gelegenheitsgedichtes inne. Als eine direkte Ansage der stattzufindenden Feierlichkeiten sei etwa kurz ein Ausschnitt dessen wie folgt zu zitieren:

Und wieder einmal schaukeln in Dorfkirchen und Kathedralen Jahrestagsglocken, wenn Streifzüge die Aufständischen in die verschneiten Kampffelder hinauslocken.

* Dr. Karol Górski, Hochschule für Logistik in Poznań, Leiter des Studiums für Fremdsprachen, ul. Estkowskiego 6, 61-755 Poznań. E-Mail: karol.gorski@wsl.com.pl

${ }^{1}$ Im Rahmen der Feierlichkeiten zum 96. Jahrestag des Ausbruchs des Großpolnischen Aufstandes 1918/19, fand Anfang 2015 die Premiere eines dies betreffend thematisierenden Films unter dem Titel: „Hiszpanka“ (Spanische Grippe) in der Regie und nach dem Drehbuch von Łukasz Barczyk statt. 
Sie brechen auf, vor dem Bazar-Hotel mit der Musik ihres Maestros in den Herzen, es kostet die Stadt den Siegesgeschmack mit den zu Vivat schießenden Gewehren...2.

Die dem Gedicht innewohnende Ladung der nationalen Gefühle und des nationalen Ruhmes der großpolnischen Gesellschaft, die an diesen Tagen, ähnlich wie seit vielen Jahren der Fall ist, völlig zum Vorschein kommen, ist eine durchaus verständliche Erscheinung und Offenbarung des immer noch lebendigen Gedächtnisses in den Herzen der Nachkommen der Aufständischen, zumindest solange die Söhne und Enkel derer noch leben und die Erinnerung daran weitertragen.

Auf der anderen Seite soll das immer mehr mythologisierte Ereignis nicht mehr den Habitus der Nationalgemeinschaft, der die Nachfahren der Aufständischen angehören, für immer und ewig einseitig bestimmen, nach dem Motto wie der Posener Germanist und namhafte Literaturwissenschaftler Hubert Orłowski von einer Zeit träumt, in welcher das Stereotyp der polnischen Wirtschaft im deutsch-polnischen, soziologischen und literarischen Diskurs den maßgebenden Ton für immer und ewig verliert.

Ähnlich ist es auch um die Belange der historischen Auseinandersetzung mit der deutsch-polnischen Geschichte der polnischen Nationalerhebung vom Jahre 1918 bestellt. Solange im wissenschaftlichen Bestreben nur das Bild des Feindes aufgedeckt wird, ferner solange nicht mit den ehemaligen Gegnern und heutzutage friedlich gesinnten Nachbarn hinter der Neiße und der Oder, sondern immer noch gegen sie die folgenden Jahrestage des Ausbruchs des Großpolnischen Aufstandes 1918/19 hierzulande begangen und gefeiert werden, solange werden auch die symbolischen Barrikaden im psychischen Gegeneinander-Empfinden zu beiden Seiten nicht aufgehoben und die Brücken zueinander nicht genügend standfest gebaut. Es ist dann nicht verwunderlich, wenn ein Posener Lied vor fast ein hundert Jahren die angebliche Rachsucht der damaligen Posener Polen total lügenhaft besagte, um das Entsetzen der deutschen Einwohner der Provinz Posen über das angeblich verbrecherische Verhalten der Aufständischen aufs Äußerste zu treiben:

...Verraten, erschlagen, viel Hunderttausend tot;

Die falschen Polenfahnen verhöhnen Deutschlands Not... ${ }^{3}$.

oder die Opfer der Posener Deutschen in Form der Überarbeitung eines bekannten Volksliedes sentimental zu rekapitulieren:

${ }^{2}$ Górski K., Na dzień 27 grudnia... (Auf den 27. Dezember...) (die zitierte Strophe übertragen ins Deutsche vom Autor selbst). Siehe den Wortlaut des ganzen Gedichtes im Polnischen im Anhang.

${ }^{3}$ Hahn W., Verdrängte sind wir. Zitiert nach Chodera J., Literatura niemiecka o Polsce w latach 1918-1939 (Deutsche Literatur über Polen in den Jahren 1918-1939), Wydawnictwo Śląsk, Katowice, 1969, S. 113. 
...O Posen, o Posen, du wunderschöne Stadt;

Darinnen liegt begraben so mancher Soldat... ${ }^{4}$.

Wenn es aber nach dem solchen Motto weiterbestehen bleiben sollte, dann schlagen weiterhin die polnischen und deutschen Glocken jede „für sich allein“ und klingeln die Klänge von falschen Maestros und in falsch „rauschenden Gesängen" gegen die gemeinsame Zukunft, wie etwa im Gedicht eines in Posen gebürtigen Nazi-Apologeten, Hans-Jürgen Nierentz, aus der Vorkriegszeit:

...Gott war in uns, als wir die Fahne trugen,

Und über uns erglomm das Firmament,

Und Glocken von den Kathedralen schlugen

Wie Herzschlag, der nach dem Wunder brennt.

So wurden wir zu Klingen und zu Klängen:

Vor lichter Zukunft, die sich offenbart,

Stehen wir gebannt in rauschenden Gesängen,

Entflammt vom Wunder großer Gegenwart...5.

Genauso konnte die von ihm erdichtete Brücke nicht zueinander führen, sondern spannte die Reihen der nachfolgenden Generationen der Deutschen auf der Brücke der Geschichte, umstellt mit Fackeln, Flammen und Glut, und zeigte den „Weg, Wille und Ziel im Blut", was lediglich eine tausendjährige, unüberbrückbare Kluft zwischen den beiden Nationen herbeiführen konnte.

\footnotetext{
„... Spannt eine Brücke von Vätern zu Enkeln sich weit.

Tausend Geschlechter im Dienst aneinandergereiht

Stehen als ragende Pfeiler und tragen sie gut,

Jeder dem andern Vater und Diener im Blut,

Jeder dem andern verpflichtet zu männlicher Tat.

Jeder dem andern: Und jeder Kamerad. ${ }^{6}$.
}

Einen ähnlichen, den Posener Polen feindlich gesinnten Tenor erhielt ein anderer, in der Stadt Poznań in der Zwischenkriegszeit lebender Aktivist der deutschen Diaspora, Valentin Polcuch, aufrecht, welcher in seinem mit Posen betitelten Gedicht der Stadt Posen zusprach, in der Vergangenheit deutsche Grenze, Brücke und zugleich Wall gewesen zu sein. Der Auslaut lässt sogar die Freude über die Rückgewinnung der ,altdeutschen“ Stadt Posen an das Deutsche Reich im September 1939 verspüren:

${ }^{4}$ Ebenda, S. 121.

${ }^{5}$ Nierentz H.-J., Gott war in uns. In: Müller-Schwanneke H., bearb. von Lüdtke, Franz: Rufer des Ostens. Hirt-Reger und v. Schroedel-Siemau. Posen, 1943, S. 176.

${ }^{6}$ Nierentz H.-J., Brücke über die Zeit. Ebenda, S. 177. 
...Grenze zu sein, Brücke und Wall zugleich, Brücke nach Osten, aber auch Weiser ins Reich. Bis dann der glückhafte Tag der Erfüllung kam, bis dich das Reich auf ewig zu sich nahm, Brücke bleibst du, Wall bleibst du und Bastei, aber die Fahne weht: Posen, dein Land ist frei!?.

Bei der literaturgeschichtlichen Auseinandersetzung mit der deutschen Dichtung, an der sich gewisse Bezüge auf den Großpolnischen Aufstand 1918/19, allerdings in Form einer gegensätzlich zur damals obliegenden Ausprägung der betreffenden Lyrik verbalisierten Botschaft, feststellen lassen, wäre die Analyse ohne Einblick in das Schicksal und das betreffende Schaffen von Sophie-Charlotte Gräfin von Schlieffen ${ }^{8}$ nicht vollständig gewesen. Ihre Familie gehörte zum deutschen, in der Provinz Posen alteingesessenen Adel an, war zum Ende des Ersten Weltkrieges Besitzerin und Verwalterin eines Landgutes in dem im Westen der Provinz Posen gelegenen Kreis Wollstein (Wolsztyn) und wollte als solche im Gegenteil zu ihren zahlreichen Volksgenossen das polnisch werdende Land nicht verlassen. Den Willen zum Bleiben brachte sie in einer poetischen Weise in einem ihrer Gedichte wie folgt zum Ausdruck:

O Du mein Heimatland, ich laß Dich nicht!

Ob viele auch den Wanderstab erfassen, -

$\mathrm{Ob}$ trübe auch der Zukunft Angesicht

Mein Pos'ner Land, ich will Dich nicht verlassen!?.

Als Einwohnerin des zum größten Teil von Polen bewohnten Dorfes blieb ihr die mit dem Ausbruch des polnischen Aufstandes zusammenhängende Umwälzung vor Ort selbstverständlich nicht erspart und die Ereignisse des Anfangs 1919 in der Region sind ihr auch zuteilgeworden. Über die Übergangszeit 1918-1919 wusste sie also Folgendes zu berichten:

7 Polcuch V., Posen. Ebenda, S. 181.

${ }^{8}$ Sophie-Charlotte Gräfin von Schlieffen wurde 1888 in Rosbitek (Rozbitek) bei Kwiltsch (Kwilcz) im Kreis Birnbaum (Międzychód) als Tochter des königlichen Offiziers Ernst von Reiche auf Rosbitek und Sophie Burggräfin und Gräfin zu Dohna-Schlodien geboren. Seit dem Tode ihres Mannes Hermann Carl Oskar Graf von Schlieffen - er war im Jahre 1915 in der Schlacht von Jaroslau (Jarosław) gefallen - lebte sie als Witwe alleine in ihrem Schloss im Dorfe Wioska bei Wollstein (Wolsztyn), wo sie als Bürgerin des polnischen Staates ein landwirtschaftliches Gut bis 1945 bewirtschaftete. Sie war eine Künstlerin, schrieb Gedichte und komponierte Musik zu ihrer eigenen Dichtung und den Gedichten ihres Mannes. Nach der Flucht der Deutschen aus Großpolen Anfang 1945 wollte sie weiterhin in Polen bleiben. Sie wurde jedoch im Frühjahr 1945 von der polnischen Staatssicherheit nach Poznań verschleppt, wo sie in einem Gefängnis im Herbst desselben Jahres ums Leben kam. Die Stätte ihrer Beisetzung bleibt bis zum heutigen Tag unbekannt.

${ }^{9}$ Schlieffen von S.-Ch., O Du mein Heimatland. In: Schlieffen-Blätter. Mitteilungen des Familienverbandes der Grafen und Herren von Schlieffen (e.V.), Nr. 2, Potsdam-Babelsberg, Juli 1939, S. 10. 
Im November 1918 kamen deutsche Soldatenräte mit der roten Armbinde in das stille Wioskaer Haus und kündeten, dass der Krieg verloren war. Am 4. Januar 1919 rückten 200 Mann der Freiheitskämpfer der polnischen Aufstandsbewegung auf den Wioskaer Hof, durchsuchten das ganze Haus nach Waffen und Munition, verhafteten die Bewohner und schlossen sie ein. Heiß tobte der Kampf, um die geliebte Heimaterde dem deutschen Vaterlande zu erhalten. In der Kreisstadt Wollstein fiel heldenmütig in erbittertem Straßenkampf Hermann Schlieffens Nachfolger, der Vorsitzende des Kreiskriegerverbandes, Hauptmann a. D. Baumeister Neßler ${ }^{10}$.

Im Nachhinein wurden aus dem Kreis viele deutsche Bürger und Bauernführer inhaftiert. Auch Gräfin von Schlieffen teilte ihr Schicksal, indem sie einige Monate in einem Gefängnis im benachbarten Grätz (Grodzisk Wlkp.) im Kreis Posen verbrachte. Sie musste sich aber einer Beliebtheit unter den hiesigen Polen erfreut haben, denn sie zeigten ihr gegenüber eine gar nicht zugemutete „Gefolgschaftstreue" auf. Die polnische Arbeiterschaft von Wioska hatte sich also sehr solidarisch benommen und in geschlossener Reihe Unterschriften gesammelt, um ihre deutsche Gutsherrin effektiv zu befreien. Es ist daher nicht verwunderlich, wenn sie in einem danach entstandenen Gedicht zu ihrer Beziehung zu den polnischen Mitbürgern in versöhnlichem Geiste u. a. schrieb:

...Haß gegen Polen soll nicht unsrer Freundschaft Losung sein,

Denn zum Hassen sind deutsche Herzen zu fromm und zu rein... ${ }^{11}$.

In ihrem Leben und Wirken handelte sie also vor allen Dingen als eine Christin. In dem Sinne wusste sie, dass ihre Aufgabe nicht Hass, sondern Liebe war. Aus diesem Wissen heraus handelte sie als Mensch und als Christ, wenn auch dies manchmal ihr persönlich größte Schwierigkeiten einbrachte. Ein namhafter, dieser Region entstammender Volksgenosse, Georg-Christoph von Unruh, der nicht nur ein bekannter Jurawissenschaftler, sondern auch leidenschaftlicher Historiker des Posener Landes war, schrieb in einem Nachruf über die Gestalt der Gräfin von Schlieffen in folgenden verehrenden Worten:

Diese Frau, welche ihrer Heimat und ihrem Volkstum aus tiefstem Herzen verpflichtet und verbunden war, welche um die großen Leistungen ihrer eigenen Vorfahren und der Deutschen im Posener Land wusste, war doch aus ihrer tiefen Menschlichkeit heraus von engem und übersteigerten Nationalgefühl völlig frei. So hat sie hinter Volk und Stand, hinter Staatsund Volksangehörigkeit immer den Menschen gesucht, wie sie auch aus ihrer tiefen Gläubigkeit heraus jeden anderen Glauben achtete und ehrte. ${ }^{12}$

${ }^{10}$ Schlieffen von S.-Ch., In: Schulz-Weitzel I., Jablone - ein Dorf in Polen. Versuch einer Rückschau auf die Besiedlung und Geschichte unserer verlorenen Heimat. Selbstverlag Ilse WeitzelSchulz. Spabrücken, 2005. S. 237.

${ }^{11}$ Schlieffenvon S.-Ch., Deutsche Brudertreue in Polen. In: Schlieffen-Blätter..., S. 12.

${ }^{12}$ Unruh von G.-Ch., In Memoriam Sophie-Charlotte Gräfin von Schlieffen. In: Deutsches Adelsarchiv, Nr. 3, 14. Jahrgang, 15. März 1958, S. 48. 
Während der Internierung durch die polnischen Aufständischen führte sie im Gefängnis zu Grätz im Zeitraum vom 4. Januar bis 15. April 1919 ein Tagebuch, in dem sie u. a. eine poetische Vision des Weges der polnischen Nation an ihre Freiheit projizierte - die wohl schönste, die je deutscher Feder entging. Im Wachtraume träumt sie also in einer schlaflosen Nacht vor sich hin und so ziehen an ihren Augen zahlreiche Bilder und Gestalten aus der Vergangenheit des deutschen Reiches vorbei. Sie gedenkt deutscher Helden, deutscher Fürsten und Kaiser, auf einmal tauchen jedoch neben Deutschen auch Polen auf, als Beobachter, Begleiter und Mitträger der deutschen Geschichte. Bald erscheinen die Polen selbst auch als Mitgestalter der Geschichte Polens. Sie kommen aus der Gruft der alten polnischen Kirche zu Grätz mit dem altverbotenen, jedoch optimistischen Lied auf ihren Lippen: „Noch ist Polen nicht verloren“. In der Vision der Verfasserin steht das ganze polnische Volk wie ein Mann auf, ,einig und stolz und stark“. Aus ihren Grüften steigen die polnischen Helden heraus: „Jan Sobieski, der edle, ritterliche Pole, Kościuszko, der wilde Rebell, Dąbrowski, der alte Opaliński, - und all die anderen Ritter“. In diesem Zuge können die großpolnischen Aufständischen allerdings auch nicht mehr ausbleiben. Die wachträumende Gräfin von Schlieffen nimmt sie folgendermaßen wahr:

...Und rund um sie herum scharen sich feldgraue Krieger in deutscher Uniform, das deutsche Gewehr über die Schulter, die deutsche Disziplin im Herzen, - das deutsche Heer polnischer Nationalität. Jan Sobieski, die Idealgestalt aller Polen, tritt vor sie hin und spricht zu ihnen: „Viereinhalb Jahre habt Ihr gekämpft für die Ehre anderer Völker, - viereinhalb Jahre habt Ihr gelitten in heiligem Nationalgefühl, - viereinhalb Jahre habt

Ihr trotz aller Bruderliebe Bruder gegen Bruder gekämpft. Mit dem Herzleid Eurer Seele habt Ihr Euch Polen errungen. Ihr habt gelitten für Euer Vaterland, jetzt kämpft für Polens Größe. Zeigt Euch würdig Eurer großen Vorfahren, seid tapfer und stolz, edel und ritterlich!

Frisch zum Kampfe! Es lebe Polen! ${ }^{13}$.

Im Rhythmus des zwölfstündigen Glockenschlages vom Turme der alten polnischen Kirche in der Stadt deklamiert die Dichterin in die sternklare Nacht ihr Gedicht hinein, die zweifelsohne eine passende Aufruf-Parole zum Aufstand für die polnischen Aufständischen hätte werden können:

Jetzt ist es Zeit, -

Frisch auf zum Streit!

Jetzt oder nie!

So künden sie

Die Schicksalsstunde,

Die Geisterstunde,

der Auferstehung des polnischen Reiches. ${ }^{14}$.

\footnotetext{
${ }^{13}$ Schlieffen von S.-Ch., Tagebuch. In: Schlieffen-Blätter..., S. 9.

${ }^{14}$ Ebenda, S. 9.
} 
Aus dem Kerker ihres Gefängnisses nimmt sie des Nachts die grollenden Donner der Geschütze der kämpferischen Auseinandersetzung zwischen den polnischen Freiheitskämpfern und den deutschen Grenzschutz-Soldaten auf. Sie betet zu dem Lenker der Schlacht, dem Vater aller Völker, für die am Kampf beteiligten Soldaten, unabhängig von deren Nationalität. Es ist kaum anzunehmen, dass die Geschichte der deutschen Literatur ein anderes, solches, gerechtes und so stark vom Evangelium und der Sorge um die zu beiden Seiten Kämpfenden geprägtes Gebet kennt, das je mit deutscher Hand verfasst worden wäre. Und dies von einer Hand, die anfänglich die Missgunst seitens der polnischen Aufständischen erfahren hat. Als eine Perle der besonderen Poetik aus der Zeit des Großpolnischen Aufstandes hat das in das lokale Gepräge hineingedichtete Gebet es sicherlich verdient, im Ganzen zitiert zu werden:

Herr, mein Gott, schaue hernieder auf unsere Heimat, auf die polnische Erde, die deutsche Erde, - und richte Du, wem dieses Land gehört. Herr, Du siehst die Glaubenstreue der Polen, die Liebe zu Deinem Evangelium, zu dem Evangelium, das einst Adalbert der Heilige aus deutschen Gauen dem polnischen Volke gebracht. Deutsche Mönche im Kloster zu Obra trugen christliche Sitte und fromme Bräuche in dieses Land, und seit diesen Tagen beten Deutsche und Polen vereint in ihrer Heimat zu Dir. Nur Du allein kannst richten ein rechtes Gericht. Herr, bleibe bei uns in der Sterbestunde unseres Volkes! Wir lassen Dich nicht, Du segnest uns denn! $!^{15}$.

Während sie betete, stieg vor ihren Augen ein Bild des möglichen Friedens, eines Einvernehmens zwischen den ehemaligen, deutschen und polnischen Kameraden, auf. Sie stellte sich zwei Bauern, den deutschen und den polnischen, in feldgrauen Uniformen vor, die gemeinsam durch die dampfende Ackerscholle, durch die polnische Erde, durch die deutsche Erde, im Frieden ihre Pflüge führen. Die Visionärin malt ein Bild aus, auf dem diejenigen, die viereinhalb Jahre Schulter an Schulter gekämpft haben, nun Scholle an Scholle das Land ihrer Väter pflügen. Hinter ihnen schreitet der große „Sämann von Nazareth“ und er streut in die Herzen der Menschen, in die polnische Erde, in die deutsche Erde, das Samenkorn seiner Liebe, der christliche Bruderliebe. Der große Gottessohn von Nazareth breitet in der Vision der Verfasserin dieser sonderbaren Eintragung in ihr Tagebuch die Hände aus und segnet den Acker, er segnet die feldgrauen Krieger, indem er sich durch den Mund der Dichterin an sie mit folgenden Worten wendet:

Weshalb hadert und streitet Ihr ums dieses Land? Heimaterde ist Gotteserde! In meines Vaters Hause sind viele Wohnungen und die darinnen wohnen, reden mancherlei Sprachen. Darum

${ }^{15}$ Ebenda, S. 10. Ein ähnliches Verhältnis zum Zusammenwirken der Andersgläubigen (Protestanten und Katholiken) im Geiste des Christentums prägte 20 Jahre später während des Zweiten Krieges das Wirken des Gründers des gegen die Ideologie des Dritten Reiches gerichteten „Kreisauer Kreises“, Helmuth James von Moltke. Siehe dazu: Brakelmann G., Helmuth James von Moltke, 1907-1945: biografia. Übersetzt aus dem Deutschen von Roman Dziergwa, mit dem Nachwort von Krzysztof Ruchniewicz. Wydawnictwo Poznańskie, 2009. 
liebet Euch untereinander, gleichwie ich Euch geliebt habe. Trachtet nach dem, das droben ist, nicht nach dem, das auf Erden ist! Denn wir haben hier keine bleibende Stätte, - die zukünftige suchen wir! Heimaterde ist Gotteserde. Von Erde bist Du genommen und zu Erde wirst Du einst werden! Ihr selbst seid nur Heimaterde - Gotteserde! Alles ist Euer, Ihr aber seid Gottes! Friede auf Erden! ${ }^{16}$.

An dieser Stelle unterbricht ein Glockenschlag den Traum und somit die dichterische Vision der Inhaftierten. Der Traum ist zwar zu Ende, die Botschaft der Dichterin hört aber damit gar nicht auf. Sie wird bestehen, wenn auch weniger in Form einer politischen Auswirkung auf die deutsch-polnischen Beziehungen in Gegenwart und Zukunft, dann sicherlich als Beispiel einer einzigartigen Dichtung, die in der Zeit der schrillen und dramatischen Auseinandersetzung zwischen den Deutschen und Polen während des Großpolnischen Aufstandes 1918/19 nach einer Form christlicher Aussöhnung suchte und als solche einen Höhepunkt der betreffenden deutschen, durchaus positiv eingefärbten Lyrik darstellt.

Viele Jahrzehnte nach den beiden Weltkriegen mussten nun aber verstrichen gewesen sein, bis der Autor der vorliegenden poetischen Auseinandersetzung als Vertreter der polnischen Nation und Nachkomme eines der damaligen polnischen „Freischärler“ in einem poetischen Duett nach dem hervorragenden Beispiel der Gräfin Sophie-Charlotte von Schlieffen mit einer deutschen Dichterin der jüngeren Generation als weiblichem Nachfahr ehemaliger Posener Deutscher, nämlich Barbara Erdmann, eine gemeinsame Front für das dichterische Besingen der Stadt Poznań aufbauen konnte, und dies ohne den Anspruch auf die Besitzexklusivität der jeweiligen Heimatgefühle der Betroffenen erheben zu wollen. Der Autor beansprucht aber im Gegensatz zu Nierentz und Polcuch in seinem ähnlich thematisierten Gedicht doch ein Recht darauf, die Präsenz einer Brücke in Poznań auf seine „polnische“, trotzdem friedliche Art und Weise wahrzunehmen und darzustellen. Dazu seien ausgewählte Ausschnitte aus seinem Gedicht zu zitieren, allerdings ohne auf die jeweiligen Nationalitäten der ehemaligen, gegenwärtigen und zukünftigen „Passanten“ hingewiesen zu haben:

...Die über die Zeiten hingeworfenen Brückenjoche verschmelzen mit Goldenen Nieten unsere Gedanken, bald ersehen wir alle, welcher wir auf dieser Brücke in voller Dankbarkeit und Achtung so gedenken.

(...)

Die buntbeleuchteten Brücken-Bögen entzücken uns

alle gegen Abend durch ihren prächtigen Glanz, sogar diejenigen, die prahlen in der fernen Welt mit ähnlichem Meisterwerk von baulicher Substanz... ${ }^{17}$.

${ }_{16}$ Ebenda, S. 10.

17 Górski K., Most Biskupa Jordana (Die Bischof-Jordan-Brücke). Das Gedicht ist eine Nachlese des von der Stadtverwaltung Poznań zum ersten Jahrestag der Einweihung der Jordan-Brücke 
Wenn die heutzutage in Poznań als in ihrem zweiten Domizil lebende Dichterin Barbara Erdmann ${ }^{18}$, der diese Stadt mittlerweile zu ihrer eigenen zweiten Heimat geworden ist, ihre Scham über die historische Hinterlassenschaft ihrer deutschen Vorahnen zum Ausdruck bringt, dann muss man diesen Akt nur mit Hochachtung begrüßen. Denn die dichterische nachfolgende Verbalisierung der Scham ist in diesem Sinne einzigartig und schafft eine gute Basis für die mögliche Erlangung einer einwandfreien Harmonie des poetischen Duetts:

\author{
...Stumm \\ unter dem Schleier \\ meines Volkes Scham \\ senke ich den Blick \\ zertreten wollen \\ die Gebeine der Mörder \\ verbrennen \\ ihre Gesinnung \\ vergraben \\ ihre wirren Köpfe...19
}

Eine solche Offenbarung der Autorin verschafft ihr sicherlich das Recht, ihre Stadt Poznań auf ihre intime Art und Weise wahrzunehmen und deren Aussehen wie folgt zu schildern:
...jünger dein Kleid
jugendlicher dein Aussehen
doch hinter der Maske
Staub der Geschichte
aus längst vergangener Zeit.“20

oder auch sich über den in die ihr zu eigen gewordene Stadt angebrochenen Frühling endgültig aufrichtig freuen zu können:

\footnotetext{
...Mütter spazieren

neuem Leben

alter Mann - allein

dankbar für Momente

dieser Begegnung

im zarten Grün
}

nach deren Versetzung und Aufbau über den Fluss Cybina in Poznań 2007 veranstalteten literarischen Wettbewerbs. Das Gedicht gewann 2008 in der Wettbewerbsaustragung eine Auszeichnung. Der Voll-Text im Deutschen befindet sich im Anhang.

${ }^{18}$ Barbara Erdmann, deutsche Autorin, Dichterin, Pädagogin, Musikerin, Therapeutin. Wohnhaft in Lennestadt (Deutschland) und in Poznań (Polen).

${ }^{19}$ Erdmann B., Blutende Erde. In: Posen - meine zweite Haut. Gedichte und Kurzgeschichten über dein und mein Leben, Verlag: Books on Demand GmbH, Norderstedt, 2008.

${ }^{20}$ Erdmann B., Posen. Ebenda. 
der Trauerweide

Warta -

Geruch einer Stadt. ${ }^{21}$.

Wenn in diesem deutsch-polnischen Duett ein nachfolgend angeführtes Rezitativ des polnischen „Mitsängers“ erklingt, dann wird sicherlich nicht gegeneinander gesungen, sondern ein Versuch vorgenommen, eine volle, sich gegenseitig ergänzende, zukunftsorientierte Harmonie zu erstellen:

...Das ist mein Poznań, die Stadt meiner Geburt, da halte ich ja seit Jahren unbiegsam so stur.

Für mich ist Poznań meine auserkorene Welt, ich bestehe nun fort unter diesem Himmelszelt. Für die ewige Brückenwache, Gott vergelte es, bin ich ab jetzt für immer bereits auserwählt $t^{22}$.

Es wäre also ein Traum vom Verfasser dieses obigen Bekenntnisses, auch einmal gemeinsam mit Barbara Erdmann in einem freundschaftlichen, deutsch-polnischen, vorurteilsfreien Unisono das Duo mit dem folgenden Wortlaut vortragen zu können:

...Das ist unser Poznań, eine Stadt vom Traum,

Die teuerste Stadt im unseres Herzens Raum.

Das ist unser Poznań, hier gibt es unser Nest, an dessen Macht und Pracht glauben wir fest da bleiben wir halt bis zum unsrer Tage Rest... ${ }^{23}$.

Demzufolge wären sicherlich auch die Vorstellung und Wunsch sowohl der „Profi-Dichterin“, als auch des „Hobby-Poeten“, dass das den beiden zu eigen gewordene Poznań nie wieder zu einer Schaubühne werden solle, auf welcher sich in Zukunft irgendeine nationale Auseinandersetzung innerhalb der europäischen, deutsch-polnischen Nachbarschaft austragen könnte.

Als Fortsetzung dieses friedlichen, poetischen Gedankenganges und einer möglichen Wunschvorstellung möge auch bald eine humanistische Idee aufblühen, dass zum 100. Jahrestag des Ausbruchs des Großpolnischen Aufstandes 1918/19, der am 27. Dezember 2018 begangen werden wird, die Feierlichkeiten zu diesem Anlass einen gemeinsamen, binationalen Charakter annehmen könnten, indem die Vertreter vom brandenburgischen Frankfurt/Oder nach Poznań kommen und der Jahrhundertfeier vor Ort beiwohnen, wobei nachfolgend die Gremien der Gesellschaft für die Aufrechterhaltung des Gedächtnisses des Großpolnischen Aufstandes 1918/19 gemeinsam mit Vertretern der Stadt aus Politik und Kultur sich nach Frankfurt begeben und dort Kränze für die im Aufstand in

${ }^{21}$ Erdmann B., Frühling in Posen. Ebenda.

${ }^{22}$ Górski K., Die Bischof-Jordan-Brücke.

${ }^{23}$ Górski K., ebenda. 
der Provinz Posen gefallenen, deutschen Grenzschutzsoldaten und Eisenbahner niederlegen würden. Eine Krönung dessen könnte dann eine deutsch-polnische, dies betreffende wissenschaftliche Konferenz zur Literatur, Geschichte und Wahrnehmung dieses Ereignisses nach 100 Jahren angesichts des gemeinsam gewordenen Europäischen Hauses und der nun so erfolgreich praktizierten, gemeinsamen, deutsch-polnischen Zugehörigkeit zur Europäischen Union werden.

\title{
ANHANG \\ Die Voll-Texte der zitierten, vom Autor der Abhandlung verfassten und ins Deutsche übertragenen Gedichte:
}

\author{
Na dzień 27 grudnia... \\ Pamięci Powstańcom Wielkopolskim Ziemi Babimojskiej i Nadnoteckiej
}

I znowu rozkołyszą się w małych kościółkach i w Katedrach rocznicowe dzwony, kiedy to wyjdą na zaśnieżone pola bitew dusz naszych Powstańców zwarte zagony. Ruszą, jak wtedy, spod Bazaru, niosąc w sercu arcypolską muzykę swego Maestra, poczuje na nowo Poznań smak zwycięstwa, wiwatem już w noc Świętego Sylwestra.

Kroczył jasny płomień grudniowego zarzewia dźwiękami Wielkopolskiej Marsylianki, dotarł wkrótce do Nowego i Starego Kramska, hen poza Babimostu kolejowe szranki. Już od przedpola Kargowej przejmowali w toku walki polską sztafetę bracia Spiralscy, za co po latach zapłacili daninę krwi w patriotyzmie tak zaparci i w boju tak dziarscy.

Wzorem Żegockiego, starosty babimojskiego, walczyli tam Białym Orłem zachwyceni, aby za drutami Sachsenhausen i Oranienburga podążyć szlakiem bezimiennych cieni. Próżno szukać dzisiaj prochów babimojskiego „Króla Polaków” w jego ojczystej ziemi, tam pamięci Teodora Spiralskiego tylko ta spiżowa tablica w kramskiego kościoła sieni.

I nad Notecią nie zabrakło Powstańcom Wielkopolskim hartu ducha i orężnego dzieła, choć hardy marynarz Mariusz Wachtel nie doczekał w Gnieźnie radości swego wesela. Posłuchaj, to z pola walki wraca Jego kompania w rytm werbla powstańczego dobosza, do Katedry spieszy, tam za duszę jej dowódcy ów nokturn Paderewskiego i żałoby msza.

Mieli przed laty w ową listopadową insurekcję swego Ordona dumni Warszawianie, mieliśmy w grudniowe Powstanie też naszą „Redutę Mettlera” i my - Poznańczanie. Dał także przykład ksiądz Mateusz Zabłocki jak cierpieć i zwyciężać pod Szubinem, tam pokazał księdzu Skorupce, jak prowadzić Polaków krzyżem, nie tylko karabinem.

Kiedy więc 27 grudnia Patron powstańczego zrywu stanie raz jeszcze na balkonie Hotelu, naszych Dziadów i Ojców obudźmy tedy, niech pod nim w dumnym szeregu staną do apelu. A potem niech dalej śpią zasłużonym, świętym snem Wielkopolskiego Powstania bohaterów i śnią o wiecznej defiladzie na Placu Wolności wśród rocznicowych sztandarów i szpalerów. 


\section{Die Bischof-Jordan-Brücke \\ (Übertragung aus dem Polnischen ins Deutsche von Karol Górski)}

Der uralte Werder zwischen Zybina und Warthe steht im würdigen Habit der steinreichen Geschichte, wer an die Thietmars Chronik nicht glauben will, soll hierher kommen noch vorm hellen Tageslichte. Wenn die erste Morgenröte gleitet von Srodka bis zum Dom über die neue des Bischofs Jordan Brücke, schaut auf sie die von Mesko auserlesene Fürstin Dubrava durch die glasgemalte Domfenster-Mosaik. Im inbrünstigen Gebet in der Goldenen Piasten-Kapelle denkt sie an die der Polanen Legende zurück, während der schläfrige Morgen hat die Brücken-Lichter in den Abgrund des Flusses schon verdrückt.

Das ist unser Poznań, eine Stadt vom Traum,

Die teuerste Stadt im unseres Herzens Raum.

Das ist unser Poznań, hier gibt es unser Nest, an dessen Macht und Pracht glauben wir fest da bleiben wir halt bis zum unsrer Tage Rest.

Die über die Zeiten hingeworfenen Brückenjoche verschmelzen mit Goldenen Nieten unsere Gedanken, bald ersehen wir alle, welcher wir auf dieser Brücke in voller Dankbarkeit und Achtung so gedenken. Vielleicht erfreuen wir uns noch des Priesters Gieburowski im silbernen Nebel verschwindender Gestalt, als er einsam den Höhepunkt seiner Sängerkunst zu erreichen vermag in der göttlichen Choranstalt. Nicht nur für ihn, sondern auch für Dich singt vor in der Abendstille das Completorium 'ne Nachtigall, ein stiller Augenblick sei Dir gegönnt, in dieser der Heiligen Jungfrau Maria Andacht- und Adventszeit.

Die buntbeleuchteten Brücken-Bögen entzücken uns alle gegen Abend durch ihren prächtigen Glanz, sogar diejenigen, die prahlen in der fernen Welt mit ähnlichem Meisterwerk von baulicher Substanz. Zwar nur an dieser Stätte, wenn der Abend mit dem Gesang der Domknaben im Hauptschiff anbricht, wenn das Glockenspiel in den Gassen vom Tumski Werder in stillem Echo erschallt in aller Zuversicht, wartet auf späten Heimkehrer mit verzeihendem Lächeln der bald Heilige Seelenhirt im weißen Kleid, welchem die Sünden und Armut der Menschen in dieser düsteren Welt taten IHM so viel Pein und Leid.

Das ist mein Poznań, die Stadt meiner Geburt, da halte ich ja seit Jahren unbiegsam so stur. Für mich ist Poznań meine auserkorene Welt, ich bestehe nun fort unter diesem Himmelszelt. Für die ewige Brückenwache, Gott vergelte es, bin ich ab jetzt für immer bereits auserwählt.

Karol Górski, Dezember 2008

\section{LITERATUR- UND ANDERE BEZUGSQUELLEN}

\section{Prosa und Lyrik}

Brakelmann G. (2009), Helmuth James von Moltke, 1907-1945: biografia. Übersetzt aus dem Deutschen von Dziergwa R., mit dem Nachwort von Ruchniewicz K. Wydawnictwo Poznańskie.

Chodera J. (1969), Literatura niemiecka o Polsce w latach 1918-1939 (Deutsche Literatur über Polen in den Jahren 1918-1939), Wydawnictwo Śląsk, Katowice. 
Erdmann B. (2008), Blutende Erde. In: Posen - meine zweite Haut. Gedichte und Kurzgeschichten über dein und mein Leben, Verlag: Books on Demand GmbH, Norderstedt.

Erdmann B. (2008), Frühling in Posen. In: Posen - meine zweite Haut. Gedichte und Kurzgeschichten über dein und mein Leben, Verlag: Books on Demand GmbH, Norderstedt.

Erdmann B. (2008), Posen. In: Posen - meine zweite Haut. Gedichte und Kurzgeschichten über dein und mein Leben, Verlag: Books on Demand GmbH, Norderstedt.

Górski K. (2008), Die Bischof-Jordan-Brücke (Manuskript in Beständen des Autors, Poznań).

Górski K. (2016), Na dzień 27 grudnia... (Manuskript in Beständen des Autors, Poznań).

Hahn W. (1969), Verdrängte sind wir. In: Literatura niemiecka o Polsce w latach 1918-1939 (Deutsche Literatur über Polen in den Jahren 1918-1939), Wydawnictwo Śląsk, Katowice.

Nierentz H.-J. (1943), Brücke über die Zeit. In: Müller-Schwanneke H., bearb. von Lüdtke F., Rufer des Ostens, Hirt-Reger und v. Schroedel-Siemau. Posen.

Nierentz H.-J. (1943), Gott war in uns. In: Müller-Schwanneke H., bearb. von Lüdtke F., Rufer des Ostens, Hirt-Reger und v. Schroedel-Siemau. Posen.

Polcuch V. Posen. In: Müller-Schwanneke H., bearb. von Lüdtke F., Rufer des Ostens, Hirt-Reger und v. Schroedel-Siemau. Posen.

Schlieffen von S.-Ch. (1939), Deutsche Brudertreue in Polen. In: Schlieffen-Blätter. Mitteilungen des Familienverbandes der Grafen und Herren von Schlieffen (e.V.), Nr. 2, Potsdam-Babelsberg, Juli.

Schlieffen von S.-Ch. (1939), O Du mein Heimatland. In: Schlieffen-Blätter. Mitteilungen des Familienverbandes der Grafen und Herren von Schlieffen (e.V.), Nr. 2, Potsdam-Babelsberg, Juli.

Schlieffen von S.-Ch. (1939), Schlieffen-Blätter, Mitteilungen des Familienverbandes der Grafen und Herren von Schlieffen (e.V.), Nr. 2, Potsdam-Babelsberg, Juli.

Schlieffen von S.-Ch. (1939), Tagebuch. In: Schlieffen-Blätter. Mitteilungen des Familienverbandes der Grafen und Herren von Schlieffen (e.V.), Nr. 2, Potsdam-Babelsberg, Juli.

Schulz-Weitzel I. (2005), Jablone - ein Dorf in Polen. Versuch einer Rückschau auf die Besiedlung und Geschichte unserer verlorenen Heimat, Selbstverlag Ilse Weitzel-Schulz. Spabrücken.

Unruh von G.-Ch. (1958), In Memoriam Sophie-Charlotte Gräfin von Schlieffen. In: Deutsches Adelsarchiv, Nr. 3, 14. Jahrgang, 15. März.

Film

„Hiszpanka“ (Spanische Grippe) w reżyserii i wg scenariusza Łukasza Barczyka (in der Regie und nach dem Drehbuch von Łukasz Barczyk), 2015.

Karol Górski

\section{POLISH-GERMAN POETIC DISCOURSE AS THE PRELUDE TO NEXT YEAR'S 100 ${ }^{\text {TH }}$ ANNIVERSARY OF OUTBREAK OF GREATER POLAND UPRISING 1918/19 IN PRUSSIAN PROVINCE OF POSEN}

(Summary)

At the end of year 2018, falls the $100^{\text {th }}$ anniversary of outbreak of Greater Poland Uprising 1918/1919 in Prussian Province of Posen. During this jubilee, in the public sphere as well as, to lesser extent in domestic circles, that sacrifice of Polish patriotism from before 100 years, will be commemorated. Official, state proceedings will focus on commemorating the participants of the 
Uprising. It will pose as an occasion to celebrate and to deposit the wreaths with white-red sashes by the memorials. Flags will be flapping, drumrolls will be beaten and oaths, to never forget the Uprising in the Province of Posen of 1918/19, will be taken in the presence of state and insurrectionary banners. Anniversary proceedings are sure to be accompanied by numerous topical papers which are bound not to slip by the German observers. Therefore, factual, scientific and even literary papers are expected to be published. Anticipated spirit of said jubilee is encapsulated in commemorative poetic works written by the Author of this paper. This study stands as the plane on which the Author engage in Polish-German poetic discourse with German poets, those who witnessed or participated in the events from before 100 years, as well as with contemporary authors associated with Province of Posen in the past or at present. Because of varied emotional charge and political tenor, this lyrical prelude to next year's $100^{\text {th }}$ anniversary of outbreak of Greater Poland Uprising 1918/1919 and its tone, in collation with its German counterparts, varies from remaining in sharp dissonance to sometimes become a duet or a perfect harmony.

Keywords: Prussian Province of Posen, Greater Poland Uprising 1918/1919, anniversary proceedings, century jubilee, the Germans of Posen, German authors, factual literature, prose, poetry, poetic discourse, nationalist lyric poetry, poems about homeland 